\title{
HPMV: Human protein mutation viewer - relating sequence mutations to protein sequence architecture and function changes
}

\author{
Westley Arthur Sherman*, ${ }^{*}$, Durga Bhavani Kuchibhatla*,\|, Vachiranee Limviphuvadh*,**, \\ Sebastian Maurer-Stroh ${ }^{*, \dagger}, \dagger$, Birgit Eisenhaber, ${ }^{*+*}$ and Frank Eisenhaber ${ }^{*}, \$, \$ \&$ \\ ${ }^{*}$ Bioinformatics Institute (BII), Agency for Science \\ Technology and Research $\left(A{ }^{*} S T A R\right)$ \\ 30 Biopolis Street \#07-01, Matrix, Singapore 138671 \\ ${ }^{\dagger}$ School of Biological Sciences (SBS) \\ Nanyang Technological University (NTU) \\ 60 Nanyang Drive, Singapore 637551 \\ ॠDepartment of Biological Sciences (DBS) \\ National University of Singapore (NUS) \\ 8 Medical Drive 4, Singapore 117597 \\ $\S_{\text {School of Computer Engineering (SCE) }}$ \\ Nanyang Technological University (NTU) \\ 50 Nanyang Drive, Singapore 637553 \\ 『westleys@bii.a-star.edu.sg \\ "durgak@bii.a-star.edu.sg \\ **vachiraneel@bii.a-star.edu.sg \\ †sebastianms@bii.a-star.edu.sg \\ †birgite@bii.a-star.edu.sg \\ \$franke@bii.a-star.edu.sg
}

Received 20 April 2015

Revised 21 August 2015

Accepted 21 August 2015

Published 26 October 2015

\begin{abstract}
Next-generation sequencing advances are rapidly expanding the number of human mutations to be analyzed for causative roles in genetic disorders. Our Human Protein Mutation Viewer (HPMV) is intended to explore the biomolecular mechanistic significance of non-synonymous human mutations in protein-coding genomic regions. The tool helps to assess whether protein mutations affect the occurrence of sequence-architectural features (globular domains, targeting signals, post-translational modification sites, etc.). As input, HPMV accepts protein mutations - as UniProt accessions with mutations (e.g. HGVS nomenclature), genome coordinates, or FASTA sequences. As output, HPMV provides an interactive cartoon showing the mutations in relation to elements of the sequence architecture. A large variety of protein sequence architectural features were selected for their particular relevance to mutation interpretation. Clicking a sequence feature in the cartoon expands a tree view of additional
\end{abstract}

This is an Open Access article published by World Scientific Publishing Company. It is distributed under the terms of the Creative Commons Attribution 3.0 (CC-BY) License. Further distribution of this work is permitted, provided the original work is properly cited. 


\title{
W. A. Sherman et al.
}

\begin{abstract}
information including multiple sequence alignments of conserved domains and a simple 3D viewer mapping the mutation to known PDB structures, if available. The cartoon is also correlated with a multiple sequence alignment of similar sequences from other organisms. In cases where a mutation is likely to have a straightforward interpretation (e.g. a point mutation disrupting a well-understood targeting signal), this interpretation is suggested. The interactive cartoon can be downloaded as standalone viewer in Java jar format to be saved and viewed later with only a standard Java runtime environment. The HPMV website is: http://hpmv.bii. a-star.edu.sg/.
\end{abstract}

Keywords: Human protein mutations; protein sequence architecture; protein function; genomic variation; clinical genome sequencing.

\section{Introduction}

Genome sequencing is rapidly becoming a routine tool for investigating medical disorders with an underlying genetic basis. ${ }^{1-3}$ Next-generation sequencing is poised to revolutionize the investigation of rare genetic disorders caused by individual mutations and, generally, the influence of specific mutations and gene variants on the fidelity of physiological functions and on pathogenesis.

The dramatic decrease in sequencing prices, coupled with technologies that allow sequencing from saliva and other easy-to-collect samples, make it feasible now to sequence affected individuals as well as their unaffected family members. In combination with lists of common variants from resources such as the 1000 genomes project $^{4}$ and PhyloTree, ${ }^{5}$ the number of possible variants can be reduced to a level where identification of a specific causative mutation is often possible.

Traditional genome-wide association studies connect phenotypic properties and disease states with genomic features just at the statistical level; yet, there is a wide range of problems and one might suggest that it will not be possible to find significant links between many genomic aberrations and any phenotype since the predictive power of these approaches is limited. ${ }^{6-9}$ It is not just the issue of largess of the analyzed subpopulations and, in some cases, even involving whole mankind might be insufficient. But for example, when the mechanistic networks involving the genes considered are complicated, their effect might be enhancing or suppressing depending on the variants of other genes participating in the system of pathways and structural associates.

Mechanistic insights into pathogenesis provide rational arguments for potential cures and, therefore, it becomes most desirable to deduce hints that indicate how protein-coding gene mutations alter gene function and modulate biomolecular pathways and subcellular structures. In the present concept of protein function, ${ }^{10,11}$ a protein sequence consists of several segments (of either globular or non-globular nature), each carrying specific structural and molecular functional properties and the overall function of the protein is a synthesis of the segments' functions. In turn, the entirety of these segments, the so-called sequence architecture, determines the range of cellular and phenotypic functions that a protein can execute.

Non-synonymous mutations alter the protein sequence and, thus, can change the molecular function of the respective segment. This effect might even include the 
disappearance or de novo emergence of the respective sequence architectural element as it is well known for targeting signals or post-translational modifications (PTMs). ${ }^{12}$ Our Human Protein Mutation Viewer (HPMV) is a tool that is designed to map reported mutations onto sequence architectural features and to explore how they might affect protein function via influencing the respective segment's properties.

There is already quite a variety of databases and programs available that support researchers in analyzing human mutations. For many specific sequence variants of interest, general and specialized databases provide valuable curated information. These include general sequence-oriented databases such as Ensembl, ${ }^{13}$ UniProt, ${ }^{14}$ NCBI RefSeq,${ }^{15}$ the wwPDB,${ }^{16}$ Pfam,${ }^{17}$ CDD,${ }^{18}$ and ProTherm ${ }^{19}$ on the one hand and disease-gene and mutation-focused databases including OMIM, ${ }^{20}$ ClinVar,${ }^{21}$ HGMD,${ }^{22}$ dbSNP,${ }^{23}$ and MITOMAP ${ }^{24}$ on the other.

Analyses attempting to relate mutations to biomolecular mechanisms are quite time consuming and there is a pressing need for software to facilitate this process. Tools such as ANNOVAR, ${ }^{25}$ SeattleSeq Annotation, ${ }^{26}$ and Ensembl's Variant Effect Predictor $^{27}$ are helpful for the first annotational step of lists of mutations as they link mutations with gene annotational features. Another class of tools including MutationTaster $^{28}$ and Mutation@A Glance ${ }^{29}$ assemble useful information from existing databases for assessing the severity of a single mutation. Similarly, Distributed Annotation System (DAS) viewers (Dasty3,${ }^{29,30}$ DASher, ${ }^{31}$ SPICE, ${ }^{32}$ and the CBS DAS Viewer $^{33}$ ) conveniently collate graphical representations from databases and servers.

Programs exemplified by SIFT, ${ }^{34,35}$ GERP,${ }^{36}$ and PolyPhen ${ }^{37}$ quantitate the likelihood that mutations affecting the coding sequence might be deleterious for protein function but without assessing possible mechanisms. Generally, these tools have a greater chance for correct prediction in regions with 3D structure since mutations that affect the required hydrophobic pattern or conserved functional residues are most likely deleterious for function. In many types of non-globular segments, it is not that clear. For mutations within 3D structures of proteins, 3D structure analysis tools such as $\mathrm{SDM}^{38}$ and FoldX ${ }^{39}$ can be used to assess their impact if the $3 \mathrm{D}$ structure of the globular domain or a homologue of the sequence studied is available.

HPMV is intended to be used downstream of tools like ANNOVAR, etc. in the analysis pipeline. When the initial stages of sequence data processing reach the level of recognizing non-synonymous mutations within protein-coding genes, the stage for applying HPMV has come. By conveniently mapping human mutations to a large variety of protein sequence architectural features and suggesting interpretations, our HPMV fills the gap between initial variant filtering and specialized analysis of individual variants. No other tool available directly compares wild-type and mutant protein sequences and assesses the effect on the existence of diverse sequence-architectural features. These features were chosen for their particular relevance to mutation interpretation based on extensive experience in computational protein function prediction and include both characteristics of globular domains and $3 \mathrm{D}$ structures as well as a variety of non-globular segments (Table 1). The tool is inspired by the computational and graphical user interface framework 
Table 1. Sequence architectural features processed by HPMV.

\begin{tabular}{|c|c|c|}
\hline Feature & Algorithm/Database & References \\
\hline Natural Variants & UniProt & UniProt Consortium $^{14}$ \\
\hline Coiled-Coil & IMP-COIL (modified) & Lupas et al. ${ }^{43}$ \\
\hline Transmembrane & Phobius & Käll et al. ${ }^{44}$ \\
\hline Signal Peptide & SignalP-3.0 & Bendtsen et al. ${ }^{45}$ \\
\hline Signal Peptide & Phobius & Käll et al. ${ }^{44}$ \\
\hline Signal Peptide & UniProt & UniProt Consortium $^{14}$ \\
\hline Transit Peptide & UniProt & UniProt Consortium $^{14}$ \\
\hline Peroxysomal Targeting & PeroPS/PTS1 (metazoan) & Neuberger et $a l .{ }^{46,47}$ \\
\hline Short Sequence Motif & UniProt & UniProt Consortium $^{14}$ \\
\hline GPI Anchor & BigPI3.2 (metazoa) & Eisenhaber et $a l .^{48-51}$ \\
\hline N-Myristoylation & MyrPS/NMT & Maurer-Stroh et al. ${ }^{52,53}$ \\
\hline Farnesylation & PrePS-FT & Maurer-Stroh et al. ${ }^{54,55}$ \\
\hline Geranylgeranylation & PrePS-GGT1 & Maurer-Stroh et al. ${ }^{54,55}$ \\
\hline Rab Geranylgeranylation & PrePS-GGT2 & Maurer-Stroh et al. ${ }^{54,55}$ \\
\hline Modified Residue & UniProt & UniProt Consortium $^{14}$ \\
\hline Low Complexity & SEG (windows 12\&25) & Wootton $^{56}$ \\
\hline Disorder & IUPred (long disorder) & Dosztányi et al. ${ }^{57}$ \\
\hline Charge runs & SAPS (charge clusters) & Brendel et $a l^{58}$ \\
\hline Pfam Domains & HMMER3 against Pfam & Finn et al. ${ }^{17}$ Eddy $^{59}$ \\
\hline PDB Structures & Ssearch against PDB & $\begin{array}{c}\text { Berman et al., }{ }^{16} \text { Farrar, }{ }^{60} \\
\text { Pearson and Lipman }\end{array}$ \\
\hline
\end{tabular}

of the ANNOTATOR suite ${ }^{40,41}$ and the ANNIE tool ${ }^{42}$ which are also publicly available. These software tools pioneered the compact graphical, cartoon-based presentation of diverse structural and functional predictions for query protein sequences. Additionally, HPMV uses the ANNOTATOR as backend engine for the sequence-based predictions and annotations. With two examples, we show how mutations can affect the occurrence and/or the properties of sequence architectural features and how such findings open a mechanistic way towards explaining the phenotypic effect of mutations.

Notably, the analysis of the biomolecular mechanistic implications of nonsynonymous mutations is complicated. Currently, it cannot be automated well since it involves many diverse manual interventions depending on the nature of the affected protein sequence segment and it also requires consultations of the specific scientific literature about related biological and medical observations. We think that having the mutations mapped to sequence architectural features and illustrating the findings with intuitively designed cartoons allows quick screening of larger sets of mutations and selecting the few that deserve more detailed attention.

\section{Results}

\subsection{HPMV functionality - input options}

The most basic input to HPMV is human protein mutations. To specify the protein mutations directly, each input line starts with a (UniProt) protein accession code 
Table 2. Mutation formats recognized by HPMV.

\begin{tabular}{ll}
\hline Mutation & \multicolumn{1}{c}{ Examples } \\
\hline Point & A123L p.A123L p.Ala123Leu \\
Nonsense & A123* p.A123* p.Ala123Ter \\
Deletion (Single) & p.A123del p.Ala123del \\
Deletion (Region) & p.A123_C124del p.Ala123_Cys124del \\
Duplication (Single) & p.A123dup p.Ala123dup \\
Duplication (Region) & p.A123_C124dup p.Ala123_Cys124dup \\
Insertion (Sequence) & p.A123_C124insQSK p.Ala123_Cys124insGlnSerLys \\
Insertion (Length) & p.A123_C124ins3 p.Ala123_Cys124ins3 \\
Deletion with Insertion (Single) & p.A123delinsWV p.Ala123delinsTrpVal \\
Deletion with Insertion (Region) & p.A123_C124delinsW p.Ala123_Cys124delinsTrp \\
Frameshift (Plain) & p.A123fs p.Ala123fs \\
Frameshift (Alternate) & p.A123Gfs p.Ala123Glyfs \\
Frameshift (with Stop) & p.A123Gfs*26 p.Ala123GlyfsTer23 \\
Extension Upstream & p.M1ext-5 p.Met1ext-5 \\
Extension Downstream & p.*110Gext*17 p.Ter110GlnextTer17 \\
\hline
\end{tabular}

followed by a set of mutation specifiers in the same input line (i.e. one or several mutations for the same protein). Several protein sequences (each being one input line) can be processed in one go. Essentially all of the HGVS mutation nomenclature ${ }^{62}$ is accepted. An example list of possible mutation formats is provided in Table 2. But HPMV also processes arbitrary labels where the first (or only) number in the label is interpreted as the sequence position. An example of accepted input lines are:

sp|Q5VW32|BROX_HUMAN p.K283E p.Cys408Tyr p.Leu175_His225del

or

Q9C000 M77?

HPMV also maps genome coordinates to UniProt entries (including alternate isoforms) on a best-effort basis — for example, when the corresponding Ensembl and UniProt records do not have major discrepancies. The mappings are parsed from the relevant UniProt and Ensembl ${ }^{13}$ data files. HPMV reads genome coordinates in a format of one genome coordinate per line and allows the user to specify column order and choose from a variety of token separators accommodating, for example, VCF format.

Other websites can also link directly to HPMV cartoons with a simple URL, for example,

http://hpmv.bii.a-star.edu.sg/SeqLink?acc=P05067\&mut=V123L.

Also, for proteins not included in the UniProt human proteome, such as proteins from other organisms, the sequence features for the cartoon can be calculated on-the-fly just from the FASTA sequence.

\subsection{HPMV functionality - output: the sequence architecture cartoon}

HPMV's main output is an interactive cartoon - provided as both an interactive (JavaScript) web page and as a standalone (Java) application. A screenshot of the 


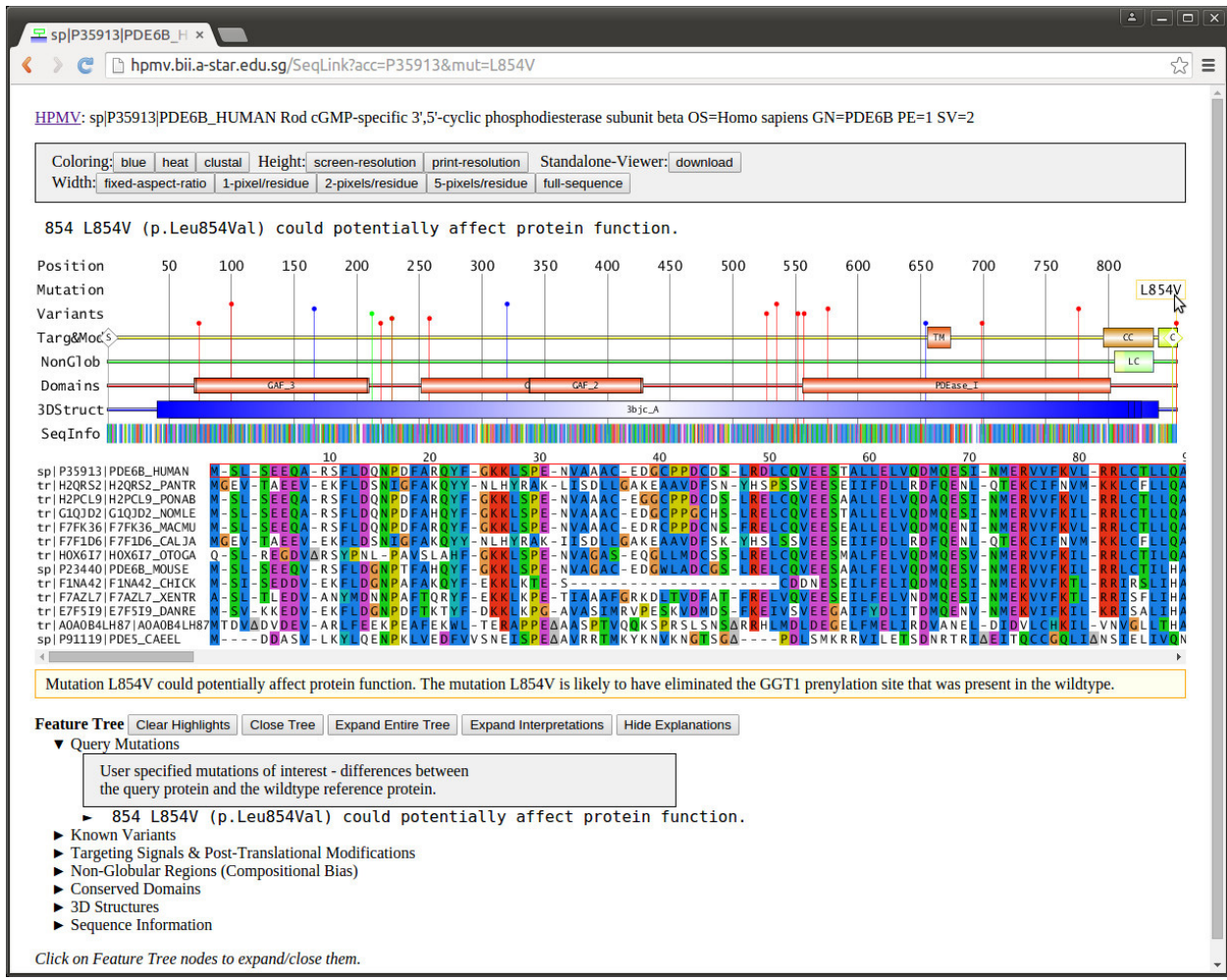

Fig. 1. Interactive cartoon for protein PDE6B along with the L854V mutation. The feature tree has been expanded to show information for the predicted C-terminal prenylation that may be disrupted by the L854V mutation. The full name of PDE6B is "Rod cGMP-specific $3^{\prime}, 5^{\prime}$-cyclic phosphodiesterase subunit beta" (UniProt: sp|P35913|PDE6B_HUMAN).

interactive web page is shown in Fig. 1. Clicking on a particular sequence feature in the cartoon image at the top of the page will provide additional information in an expandable tree format at the bottom of the page. For sequences that contain conserved globular domains (e.g. from Pfam), a multiple sequence alignment for the domain can be displayed. For sequences that have a mapping to known 3D structures, a simple wire frame view of the structure can also be displayed showing the location of mutation(s) on the structure. Directly below the cartoon image is a multiple sequence alignment of similar sequences from other organisms. In cases, where a mutation is likely to have straightforward interpretation, this interpretation is suggested in a highlighted text box just above the expandable feature tree.

Designing a graphical representation of sequence features that occur along a sequence presents significant challenges. On one hand, laying out everything in a single band can become very crowded causing essential information to become buried under other elements. On the other hand, giving each type of sequence feature its own band can result in an image that is too large for many uses. By designing the HPMV 
Table 3. Color code for functional effect of mutations.

\begin{tabular}{lcl}
\hline Functional effect & Color & \multicolumn{1}{c}{ Meaning } \\
\hline LIKELY & Red & $\ldots$. is likely to affect... \\
MORE LIKELY & Orange & $\ldots$. could potentially affect... \\
LESS LIKELY & Cyan & $\ldots$. is less likely to affect... \\
UNLIKELY & Blue & $\ldots$. is unlikely to affect... \\
UNCERTAIN & Green & $\ldots$.has uncertain/unknown effect... \\
NONE & Black & $\ldots$. .has not been interpreted... \\
\hline
\end{tabular}

cartoon around a carefully chosen set of six bands (described below), we hope to have found an optimal medium.

Briefly, the first band, "Mutation", shows the user specified mutations of interest - the difference between the query protein and the wildtype reference protein. The color code suggests the likelihood to affect the respective functional feature (Table 3).

The second band, "Variants", displays natural variants (red: specific annotation - usually disease variant; blue: dbSNP variant — without additional annotation; green: no information available).

The third band, "Targ\&Mod", visualizes certain non-globular segments such as targeting signals, PTMs, and coiled-coil and transmembrane segments. The fourth band, "NonGlob", shows other non-globular features relating to sequence composition including charge clusters, low complexity regions, and regions predicted to be disordered. The fifth band, globular "Domains", indicates matches within the sequence to known conserved domains. Finally, the sixth band, "3DStruct", shows any PDB structures with significant sequence similarity to the query sequence (highest E-value being overlayed on top).

In the case of multi-sequence input, the user is first shown the cartoons generated for all sequences in one view (Fig. 2). Then, additional information is accessible by opening separate windows for each sequence of interest that can be navigated as in the case of single protein input (see also Supplementary Figs. S1 and S2 provided as additional files).

The cartoon image can be exported in PNG format. A key feature of this export is that the width/horizontal-resolution of the image can be specified in terms of pixels per residue. This allows cartoons for sequences of different length to all be printed with the same scale.

\subsection{HPMV functionality - output: the sequence feature tree}

Details for the sequence features shown in the top cartoon image can be accessed by expanding the Feature Tree in the lower panel (Fig. 1). The first branching level of the tree corresponds to the six bands in the cartoon image. The next branching level shows sequence features within that band followed by a branch level for the individual algorithms that predicted the feature. The final branch level shows details of 


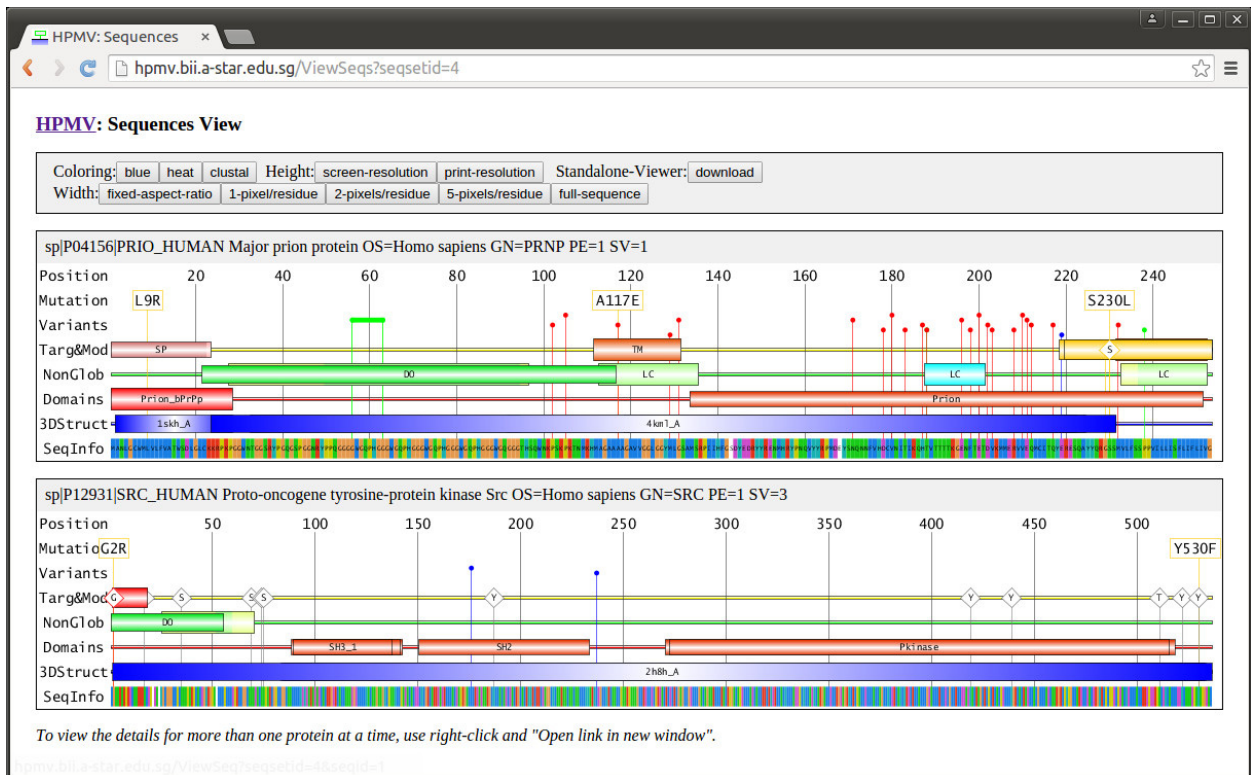

Fig. 2. Interactive cartoon for multiple protein input each having several mutations. This screenshot shows the primary output, the cartoons, for the input lines "sp|P04156|PRIO_HUMAN L9R A117E S230L" and "sp|P12931|SRC_HUMAN G2R Y530F". The detailed feature tree can be seen individually for each protein by opening a new window. The respective views are captured in the Supplementary Figs. S1 and S2 provided as additional files.

the algorithm predictions, such as scores, and any additional information associated with the function prediction. The feature tree can be sequentially opened and closed but also be fully opened and closed by a one-button click.

For conserved domains, clicking the "MSA" button will show a multiple sequence alignment (MSA) for that domain. To note, there is no automated jump between the sequence coordinate in the cartoon and in the MSA (this is a browser version issue; so, HPMV is compatible with many older versions). The problem is caused by gaps/ insertions in the alignment in comparison with the query sequence. By setting the width of the cartoon to "full-sequence", approximate alignment can be achieved.

For 3D structures, clicking the "3D" button will show a simple rotatable wireframe representation of the PDB structure - with labels for the positions of any mutations that happen to overlap the structure.

An interpretation of the effect of the mutation is suggested as part of the feature tree. The comments hint on the emergence or disappearance of a feature as a result of the mutation as well as possible changes of scores. In the case of mutations hitting globular domains with known structures, the structure visualization together with the MSA can be used to judge whether the mutation affects the hydrophobic pattern typical for the fold or any of the conserved functional sequence regions. 


\subsection{HPMV functionality - output: alignment and sequence conservation}

The conservation MSA for the middle panel of the interactive cartoon was constructed by using ggsearch ${ }^{60,61}$ to find the best global alignment match (where it existed) in each proteome from a diverse range of organisms. Then, these sequences were aligned with mafft-ginsi. ${ }^{63}$ All the MSAs can be colored according to sequence conservation (blue or heat map) or the standard Clustal color scheme.

\subsection{HPMV functionality - output: sequence feature prediction}

The algorithms used for predicting the cartoon's sequence features are listed in Table 1. They are either developed within our research division (for example, for lipid $\mathrm{PTMs}^{50}$ ) or they are contributed by the academic community. The specific prediction scores from the algorithms can be accessed by expanding the tree view of the sequence feature information. The computations are run on a cluster managed by the PBS/torque cluster management system. For proteins that are part of the UniProt human proteome, sequence features and conservation MSAs are precalculated.

For conserved domains and PDB structures, redundancy is a major challenge. For the conserved domains, using only Pfam domains ${ }^{17}$ rather than all domains in CDD was judged to be the best solution. For PDB structures, redundancy was reduced by using a filtering scheme inspired by PISCES. ${ }^{64}$

HPMV also imports certain features from the UniProt annotations as listed in Table 1. UniProt gives the position of the features relative to the canonical isoforms so HPMV performs its own mapping to also show the correct locations of the features on the alternate isoforms.

\subsection{HPMV implementation: HTML4-compliant webserver version and the standalone Java application}

Most of the protein features that HPMV shows and analyzes are obtained from running feature prediction algorithms on the protein as well as on mutant sequences. The protein features for the (wild type) UniProt sequence are pre-calculated and, for interpretations, the feature prediction algorithms are run again for the mutant sequence when it is submitted to the HPMV website by a user. Protein function is likely to be affected if the mutation affects whether the protein feature is predicted to occur in the wild type versus the mutant sequence.

The HPMV server is one of BII's WWW functionalities backed by 512 core compute cluster. When a user submits mutation to the web server, a compute client is submitted to the job queue in the cluster. The compute client runs the prediction algorithms, parses them and packages them up as Java objects, connects back to the web server, and sends back the Java objects using the java serializable protocol. The HPMV web server combines the mutation predictions with its precalculated features for the wild type sequence (stored in custom indexed data files on a solid-state 


\section{W. A. Sherman et al.}

drive) - and then provides the interpreted data to the user as its interactive cartoon. Ideally, as HPMV becomes widely used, the biological database updates will be synchronized with UniProt releases (essentially, every four weeks). But, for our own use, we will need to update the database at least every six months.

In addition to the HTML4-compliant webserver version, the interactive cartoon is also available as a standalone Java application. The interactive cartoon can contact the HPMV web server for data interactively but it can also be embedded with the data for a user-specified set of protein mutations (using Java serialization and jar file technologies). This embedding allows the interactive cartoons to be saved and viewed later using only the standard Java Runtime Environment. The standalone Java application has been verified to run correctly on the three major operating systems: Windows, MacOS, and Linux.

The standalone application also has some extra interactive features for displaying the MSAs. The conservation MSA scrolling is coupled to the gray bar at the top of the cartoon image — showing where the displayed alignment falls relative to the cartoon. Selecting a particular sequence in the MSA shows the numbering for that sequence. Sliders provide more compact and convenient views of the MSA. The vertical slider incrementally removes redundant sequences from the MSA. The horizontal slider incrementally compresses large gaps in the MSA.

\subsection{HPMV application: Variant L854 V in protein PDE6B}

HPMV includes a set of feature prediction algorithms that were developed previously within our research group. The following examples highlight the utility of these algorithms for human mutation interpretation. The mutation shown in Fig. 1, L854V in protein PDE6B, is known as a cause of Retinitis Pigmentosa 40 (RP40). ${ }^{65}$ It maps into the non-globular segment (840-854) responsible for S-geranylgeranylation. This mutation is predicted by the published PrePS webserver ${ }^{54}$ to alter an S-geranylgeranyl PTM motif by switching its specificity to S-farnesylation instead (the corresponding interactive cartoon can be found on the HPMV website as both a web page link and a downloadable jar file). The specific lipid anchor type may play a role in proper localization and complex formation of PDE6B at the membrane. ${ }^{66}$

\subsection{HPMV application: Variant $\triangle S 26$ in protein XRPQ}

The single residue deletion $\Delta \mathrm{S} 6$ maps into the non-globular segment 1-17 of XRP2 (sp|O75695|) responsible for N-terminal myristoylation (Fig. 3). This mutation is predicted by the published MyrPS/NMT webserver as abolishing myristoylation. It is thought that the failure to myristoylate the protein at Gly-2 will lead to weak interaction with intracellular membranes and to inefficient palmitoylation at Cys-3 (see the HPMV website for a downloadable jar file or link to the corresponding interactive cartoon). Thus, improper subcellular localization ${ }^{67}$ appears to be the biomolecular mechanism that causes Retinitis Pigmentosa 2 (RP2) in this case. ${ }^{67-70}$ 


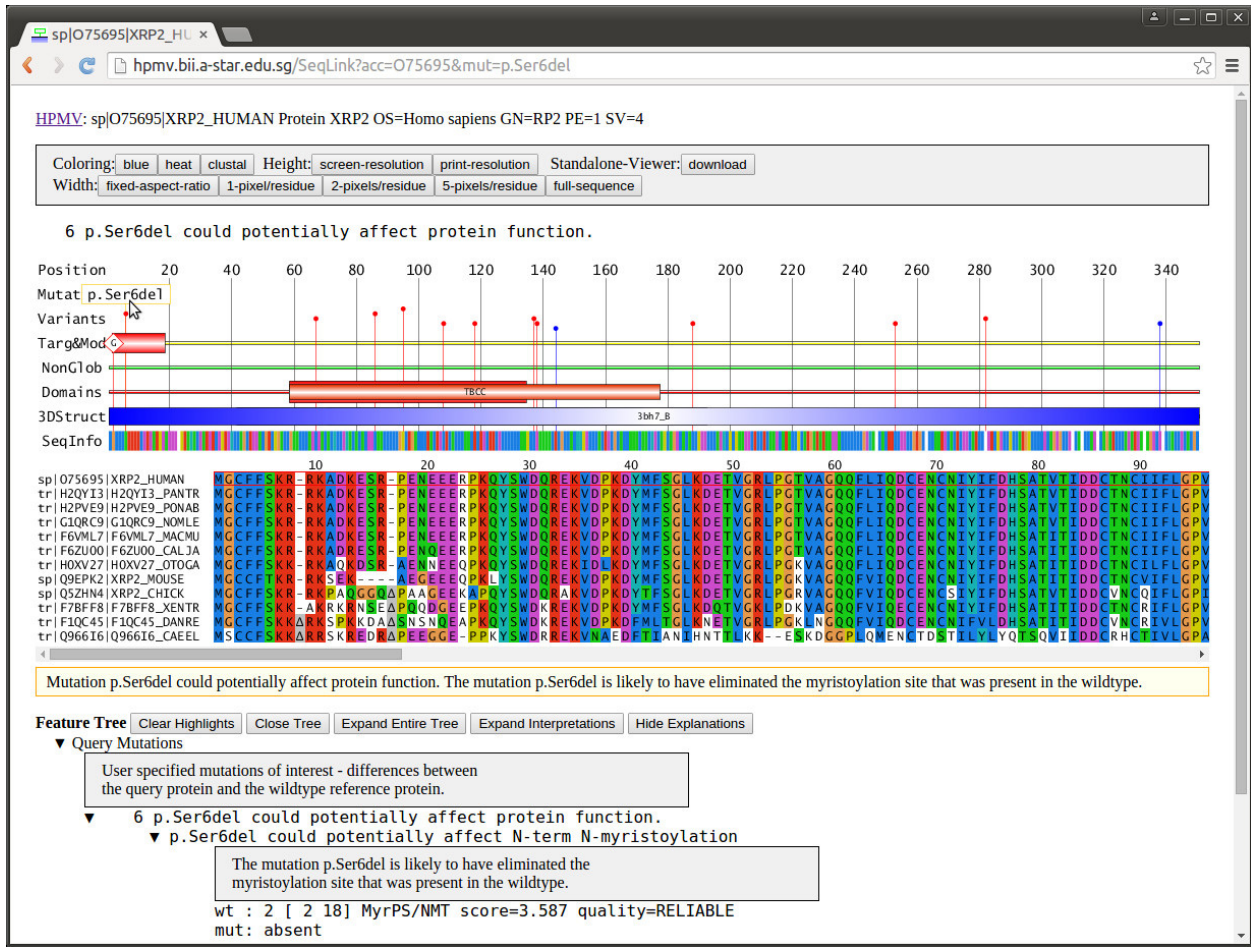

Fig. 3. Interactive cartoon for protein XRP2 along with the variant $\Delta \mathrm{S} 26$. The feature tree has been expanded to show information for the predicted N-terminal myristoylation that is disrupted by the $\Delta \mathrm{S} 26$ mutation. The protein XRP2 (O75695) acts as a GTPase-activating protein (GAP) involved in intracellular protein trafficking and protein localization. Thus, the lipid anchor is important for correct functioning.

\section{Discussion}

HPMV is intended to fill a niche between initial coarse filtering of the full list of variants generated by next-generation sequencing and detailed manual analysis of one or two variants of particular interest. The idea is that an investigator can submit a few dozen variants to HPMV and by a quick and easy inspection of each cartoon arrive at a short list of one or two variants of particular interest for further study.

As general biological databases become increasingly detailed, there is an emerging need to present the most relevant information for a particular task. By focusing specifically on the task of human mutation interpretation and by leveraging our extensive expertise in computational protein function prediction, HPMV is able to present a selection of sequence features where the likely impact of a mutation can be assessed with only a quick glance at the cartoon. These sequence feature prediction algorithms are run uniformly across all sequences insuring a high level of consistency.

In the future, we intend to continue to enrich the sequence architectural features and interpretations that are provided by HPMV. High on our list are a more 


\section{W. A. Sherman et al.}

complete set of transmembrane predictors, ${ }^{71-74}$ the concept of simple and complex ${ }^{75}$ as well as enrichment with many more short motifs known to have functional significance ( such as Huf-Zinc ${ }^{76}$ ). We also intend to develop, with guidance from our users, capabilities that allow HPMV to be more easily integrated into clinical genetics workflows.

\section{Conclusions}

The HPMV is an efficient software tool to relate human protein sequence mutations to sequence architectural features and to study their influence on mechanistic, segment-specific functional properties of proteins.

\section{Acknowledgments}

This work was supported by the Bioinformatics Institute, A*STAR, Singapore, especially by the grants IAF311010 ("Integrated genomics Platform") and IMaGIN. The authors thank Yong Tai Pang for technical support in setting up and in maintaining the web server.

\section{References}

1. Eisenhaber F, A decade after the first full human genome sequencing: When will we understand our own genome? J Bioinform Comput Biol 10:1271001, 2012.

2. Kuznetsov V, Lee HK, Maurer-Stroh S, Molnar MJ, Pongor S, Eisenhaber B, Eisenhaber F, How bioinformatics influences health informatics: Usage of biomolecular sequences, expression profiles and automated microscopic image analyses for clinical needs and public health, Health Inf Sci Syst 1:2, 2013.

3. Eisenhaber F, Sung WK, Wong L, The 24th International Conference on Genome Informatics, GIW2013, in Singapore, J Bioinform Comput Biol 11:1302003, 2013.

4. Abecasis GR, Auton A, Brooks LD, DePristo MA, Durbin RM, Handsaker RE, Kang HM, Marth GT, McVean GA, An integrated map of genetic variation from 1,092 human genomes, Nature 491:56-65, 2012.

5. van OM, Kayser M, Updated comprehensive phylogenetic tree of global human mitochondrial DNA variation, Hum Mutat 30:E386-E394, 2009.

6. Altmuller J, Palmer LJ, Fischer G, Scherb H, Wjst M, Genomewide scans of complex human diseases: True linkage is hard to find, Am J Hum Genet 69:936-950, 2001.

7. Pearson TA, Manolio TA, How to interpret a genome-wide association study, JAMA 299:1335-1344, 2008.

8. Apalasamy YD, Mohamed Z, Obesity and genomics: Role of technology in unraveling the complex genetic architecture of obesity, Hum Genet 134:361-374, 2015.

9. Auer PL, Lettre G, Rare variant association studies: Considerations, challenges and opportunities, Genome Med 7:16, 2015.

10. Bork P, Dandekar T, az-Lazcoz Y, Eisenhaber F, Huynen M, Yuan Y, Predicting function: From genes to genomes and back, J Mol Biol 283:707-725, 1998.

11. Eisenhaber F, Prediction of protein function: Two basic concepts and one practical recipe, in Eisenhaber F (ed.), Discovering Biomolecular Mechanisms with Computational Biology, 1st Edition, Chapter 3, Landes Biosciences and Eurekah.com, Georgetown, pp. 39-54, 2006. 
12. Neuberger G, Kunze M, Eisenhaber F, Berger J, Hartig A, Brocard C, Hidden localization motifs: Naturally occurring peroxisomal targeting signals in non-peroxisomal proteins, Genome Biol 5:R97, 2004.

13. Flicek P, Amode MR, Barrell D, Beal K, Billis K, Brent S, Carvalho-Silva D, Clapham P, Coates G, Fitzgerald S, Gil L, Giron CG, Gordon L, Hourlier T, Hunt S, Johnson N, Juettemann T, Kahari AK, Keenan S, Kulesha E, Martin FJ, Maurel T, McLaren WM, Murphy DN, Nag R, Overduin B, Pignatelli M, Pritchard B, Pritchard E, Riat HS, Ruffier M, Sheppard D, Taylor K, Thormann A, Trevanion SJ, Vullo A, Wilder SP, Wilson M, Zadissa A, Aken BL, Birney E, Cunningham F, Harrow J, Herrero J, Hubbard TJ, Kinsella R, Muffato M, Parker A, Spudich G, Yates A, Zerbino DR, Searle SM, Ensembl 2014, Nucl Acids Res 42:D749-D755, 2014.

14. UniProt Consortium, Update on activities at the Universal Protein Resource (UniProt) in 2013, Nucl Acids Res 41:D43-D47, 2013.

15. Pruitt KD, Tatusova T, Brown GR, Maglott DR, NCBI Reference Sequences (RefSeq): Current status, new features and genome annotation policy, Nucl Acids Res 40: D130-D135, 2012.

16. Berman H, Henrick K, Nakamura H, Announcing the worldwide Protein Data Bank, Nat Struct Biol 10:980, 2003.

17. Finn RD, Bateman A, Clements J, Coggill P, Eberhardt RY, Eddy SR, Heger A, Hetherington K, Holm L, Mistry J, Sonnhammer EL, Tate J, Punta M, Pfam: The protein families database, Nucl Acids Res 42:D222-D230, 2014.

18. Marchler-Bauer A, Zheng C, Chitsaz F, Derbyshire MK, Geer LY, Geer RC, Gonzales NR, Gwadz M, Hurwitz DI, Lanczycki CJ, Lu F, Lu S, Marchler GH, Song JS, Thanki N, Yamashita RA, Zhang D, Bryant SH, CDD: Conserved domains and protein threedimensional structure, Nucl Acids Res 41:D348-D352, 2013.

19. Kumar MD, Bava KA, Gromiha MM, Prabakaran P, Kitajima K, Uedaira H, Sarai A, ProTherm and ProNIT: Thermodynamic databases for proteins and protein-nucleic acid interactions, Nucl Acids Res 34:D204-D206, 2006.

20. Amberger J, Bocchini C, Hamosh A, A new face and new challenges for Online Mendelian Inheritance in Man (OMIM(R)), Hum Mutat 32:564-567, 2011.

21. Landrum MJ, Lee JM, Riley GR, Jang W, Rubinstein WS, Church DM, Maglott DR, ClinVar: Public archive of relationships among sequence variation and human phenotype, Nucl Acids Res 42:D980-D985, 2014.

22. Stenson PD, Ball EV, Mort M, Phillips AD, Shaw K, Cooper DN, The Human Gene Mutation Database (HGMD) and its exploitation in the fields of personalized genomics and molecular evolution, Curr Protoc Bioinformatics Chapter 1:Unit1, 2012.

23. Sherry ST, Ward MH, Kholodov M, Baker J, Phan L, Smigielski EM, Sirotkin K, dbSNP: The NCBI database of genetic variation, Nucl Acids Res 29:308-311, 2001.

24. Ruiz-Pesini E, Lott MT, Procaccio V, Poole JC, Brandon MC, Mishmar D, Yi C, Kreuziger J, Baldi P, Wallace DC, An enhanced MITOMAP with a global mtDNA mutational phylogeny, Nucl Acids Res 35:D823-D828, 2007.

25. Wang K, Li M, Hakonarson H, ANNOVAR: Functional annotation of genetic variants from high-throughput sequencing data, Nucl Acids Res 38:e164, 2010.

26. Ng SB, Turner EH, Robertson PD, Flygare SD, Bigham AW, Lee C, Shaffer T, Wong M, Bhattacharjee A, Eichler EE, Bamshad M, Nickerson DA, Shendure J, Targeted capture and massively parallel sequencing of 12 human exomes, Nature 461:272-276, 2009.

27. McLaren W, Pritchard B, Rios D, Chen Y, Flicek P, Cunningham F, Deriving the consequences of genomic variants with the Ensembl API and SNP effect predictor, Bioinformatics 26:2069-2070, 2010. 
28. Schwarz JM, Rodelsperger C, Schuelke M, Seelow D, MutationTaster evaluates diseasecausing potential of sequence alterations, Nat Methods 7:575-576, 2010.

29. Hijikata A, Raju R, Keerthikumar S, Ramabadran S, Balakrishnan L, Ramadoss SK, Pandey A, Mohan S, Ohara O, Mutation@A Glance: An integrative web application for analysing mutations from human genetic diseases, DNA Res 17:197-208, 2010.

30. Villaveces JM, Jimenez RC, Garcia LJ, Salazar GA, Gel B, Mulder N, Martin M, Garcia A, Hermjakob H, Dasty3, a WEB framework for DAS, Bioinformatics 27:2616-2617, 2011.

31. Messina DN, Sonnhammer EL, DASher: A stand-alone protein sequence client for DAS, the distributed annotation system, Bioinformatics 25:1333-1334, 2009.

32. Prlic A, Down TA, Hubbard TJ, Adding some SPICE to DAS, Bioinformatics 21(suppl 2):ii40-ii41, 2005.

33. Olason PI, Integrating protein annotation resources through the Distributed Annotation System, Nucl Acids Res 33:W468-W470, 2005.

34. Ng PC, Henikoff S, Predicting deleterious amino acid substitutions, Genome Res 11:863874, 2001.

35. Sim NL, Kumar P, Hu J, Henikoff S, Schneider G, Ng PC, SIFT web server: Predicting effects of amino acid substitutions on proteins, Nucl Acids Res 40:W452-W457, 2012.

36. Cooper GM, Stone EA, Asimenos G, Green ED, Batzoglou S, Sidow A, Distribution and intensity of constraint in mammalian genomic sequence, Genome Res 15:901-913, 2005 .

37. Adzhubei IA, Schmidt S, Peshkin L, Ramensky VE, Gerasimova A, Bork P, Kondrashov AS, Sunyaev SR, A method and server for predicting damaging missense mutations, Nat Methods 7:248-249, 2010.

38. Worth CL, Bickerton GR, Schreyer A, Forman JR, Cheng TM, Lee S, Gong S, Burke DF, Blundell TL, A structural bioinformatics approach to the analysis of nonsynonymous single nucleotide polymorphisms (nsSNPs) and their relation to disease, J Bioinform Comput Biol 5:1297-1318, 2007.

39. Schymkowitz J, Borg J, Stricher F, Nys R, Rousseau F, Serrano L, The FoldX web server: An online force field, Nucl Acids Res 33:W382-W388, 2005.

40. Schneider G, Wildpaner M, Sirota FL, Maurer-Stroh S, Eisenhaber B, Eisenhaber F, Integrated tools for biomolecular sequence-based function prediction as exemplified by the ANNOTATOR software environment, Methods Mol Biol 609:257-267, 2010.

41. Schneider G, Sherman W, Kuchibhatla D, Ooi HS, Sirota FL, Maurer-Stroh S, Eisenhaber B, Eisenhaber F, Protein sequence-structure-function-network links discovered with the ANNOTATOR software suite: Application to Elys/Mel-28, in Trajanoski Z (ed.), Computational Medicine, Springer, Vienna, pp. 111-143, 2012.

42. Ooi HS, Kwo CY, Wildpaner M, Sirota FL, Eisenhaber B, Maurer-Stroh S, Wong WC, Schleiffer A, Eisenhaber F, Schneider G, ANNIE: Integrated de novo protein sequence annotation, Nucl Acids Res 37:W435-W440, 2009.

43. Lupas A, Van DM, Stock J, Predicting coiled coils from protein sequences, Science 252:1162-1164, 1991.

44. Kall L, Krogh A, Sonnhammer EL, A combined transmembrane topology and signal peptide prediction method, J Mol Biol 338:1027-1036, 2004.

45. Bendtsen JD, Nielsen H, von HG, Brunak S, Improved prediction of signal peptides: SignalP 3.0, J Mol Biol 340:783-795, 2004.

46. Neuberger G, Maurer-Stroh S, Eisenhaber B, Hartig A, Eisenhaber F, Prediction of peroxisomal targeting signal 1 containing proteins from amino acid sequence, $\mathrm{J} \mathrm{Mol} \mathrm{Biol}$ 328:581-592, 2003. 
47. Neuberger G, Maurer-Stroh S, Eisenhaber B, Hartig A, Eisenhaber F, Motif refinement of the peroxisomal targeting signal 1 and evaluation of taxon-specific differences, $\mathrm{J} \mathrm{Mol} \mathrm{Biol}$ 328:567-579, 2003.

48. Eisenhaber B, Bork P, Eisenhaber F, Prediction of potential GPI-modification sites in proprotein sequences, J Mol Biol 292:741-758, 1999.

49. Eisenhaber B, Wildpaner M, Schultz CJ, Borner GH, Dupree P, Eisenhaber F, Glycosylphosphatidylinositol lipid anchoring of plant proteins. Sensitive prediction from sequence- and genome-wide studies for Arabidopsis and rice, Plant Physiol 133:1691-1701, 2003.

50. Eisenhaber B, Eisenhaber F, Maurer-Stroh S, Neuberger G, Prediction of sequence signals for lipid post-translational modifications: Insights from case studies, Proteomics 4:16141625, 2004.

51. Eisenhaber B, Schneider G, Wildpaner M, Eisenhaber F, A sensitive predictor for potential GPI lipid modification sites in fungal protein sequences and its application to genome-wide studies for Aspergillus nidulans, Candida albicans, Neurospora crassa, Saccharomyces cerevisiae and Schizosaccharomyces pombe, J Mol Biol 337:243-253, 2004.

52. Maurer-Stroh S, Eisenhaber B, Eisenhaber F, N-terminal N-myristoylation of proteins: Prediction of substrate proteins from amino acid sequence, J Mol Biol 317:541-557, 2002.

53. Maurer-Stroh S, Eisenhaber B, Eisenhaber F, N-terminal N-myristoylation of proteins: Refinement of the sequence motif and its taxon-specific differences, J Mol Biol 317:523$540,2002$.

54. Maurer-Stroh S, Eisenhaber F, Refinement and prediction of protein prenylation motifs, Genome Biol 6:R55, 2005.

55. Maurer-Stroh S, Koranda M, Benetka W, Schneider G, Sirota FL, Eisenhaber F, Towards complete sets of farnesylated and geranylgeranylated proteins, PLoS Comput Biol 3:e66, 2007.

56. Wootton JC, Non-globular domains in protein sequences: Automated segmentation using complexity measures, Comput Chem 18:269-285, 1994.

57. Dosztanyi Z, Csizmok V, Tompa P, Simon I, The pairwise energy content estimated from amino acid composition discriminates between folded and intrinsically unstructured proteins, J Mol Biol 347:827-839, 2005.

58. Brendel V, Bucher P, Nourbakhsh IR, Blaisdell BE, Karlin S, Methods and algorithms for statistical analysis of protein sequences, Proc Natl Acad Sci U S A 89:2002-2006, 1992.

59. Eddy SR, Accelerated profile HMM searches, PLoS Comput Biol 7:e1002195, 2011.

60. Farrar M, Striped Smith-Waterman speeds database searches six times over other SIMD implementations, Bioinformatics 23:156-161, 2007.

61. Pearson WR, Lipman DJ, Improved tools for biological sequence comparison, Proc Natl Acad Sci U S A 85:2444-2448, 1988.

62. Taschner PE, den Dunnen JT, Describing structural changes by extending HGVS sequence variation nomenclature, Hum Mutat 32:507-511, 2011.

63. Katoh K, Standley DM, MAFFT multiple sequence alignment software version 7: Improvements in performance and usability, Mol Biol Evol 30:772-780, 2013.

64. Wang G, Dunbrack RL Jr., PISCES: A protein sequence culling server, Bioinformatics 19:1589-1591, 2003.

65. Veske A, Orth U, Rüther K, Zrenner E, Rosenberg T, Baehr W and Gal A, Mutations in the gene for the $\beta$-subunit of rod photoreceptor cGMP-specific phosphodiesterase (PDEB) in patients with retinal dystrophies and dysfunctions, in Anderson RE, Laail MM, Hollyfield JG (eds.), Degenerative Disease of the Retina, Chapter 35, Plenum Press, New York and London, pp. 313-322, 2015. 
66. Anant JS, Ong OC, Xie HY, Clarke S, O'Brien PJ, Fung BK, In vivo differential prenylation of retinal cyclic GMP phosphodiesterase catalytic subunits, J Biol Chem 267:687-690, 1992.

67. Chapple JP, Hardcastle AJ, Grayson C, Spackman LA, Willison KR, Cheetham ME, Mutations in the N-terminus of the X-linked retinitis pigmentosa protein RP2 interfere with the normal targeting of the protein to the plasma membrane, Hum Mol Genet 9:1919-1926, 2000.

68. Bartolini F, Bhamidipati A, Thomas S, Schwahn U, Lewis SA, Cowan NJ, Functional overlap between retinitis pigmentosa 2 protein and the tubulin-specific chaperone cofactor C, J Biol Chem 277:14629-14634, 2002.

69. Rosenberg T, Schwahn U, Feil S, Berger W, Genotype-phenotype correlation in X-linked retinitis pigmentosa 2 (RP2), Ophthalmic Genet 20:161-172, 1999.

70. Schwahn U, Lenzner S, Dong J, Feil S, Hinzmann B, van DG, Kirschner R, Hemberger M, Bergen AA, Rosenberg T, Pinckers AJ, Fundele R, Rosenthal A, Cremers FP, Ropers $\mathrm{HH}$, Berger W, Positional cloning of the gene for X-linked retinitis pigmentosa 2, Nat Genet 19:327-332, 1998.

71. Cserzo M, Eisenhaber F, Eisenhaber B, Simon I, On filtering false positive transmembrane protein predictions, Protein Eng 15:745-752, 2002.

72. Krogh A, Larsson B, von HG, Sonnhammer EL, Predicting transmembrane protein topology with a hidden Markov model: Application to complete genomes, J Mol Biol 305:567-580, 2001.

73. Tusnady GE, Simon I, Principles governing amino acid composition of integral membrane proteins: Application to topology prediction, J Mol Biol 283:489-506, 1998.

74. von Heijne G, Membrane protein structure prediction. Hydrophobicity analysis and the positive-inside rule, J Mol Biol 225:487-494, 1992.

75. Wong WC, Maurer-Stroh S, Schneider G, Eisenhaber F, Transmembrane helix: Simple or complex, Nucl Acids Res 40:W370-W375, 2012.

76. Maurer-Stroh S, Gao H, Han H, Baeten L, Schymkowitz J, Rousseau F, Zhang L, Eisenhaber F, Motif discovery with data mining in 3D protein structure databases: Discovery, validation and prediction of the U-shape zinc binding ("Huf-Zinc") motif, J Bioinform Comput Biol 11:1340008, 2013.

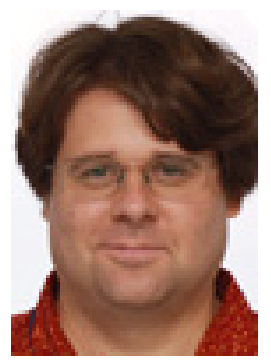

Westley Arthur Sherman did his BSc in biology at the MIT in Cambridge/Boston (1995) and the PhD in biological chemistry at the University of Michigan (2002). This was followed by a postdoctoral position at the Keck Graduate Institute (Clermont Colleges/California) and, since 2010, he has joined the Bioinformatics Institute where he is currently working as research scientist. His scientific interest is focused on the analysis of biomolecular sequences and the interpretation of mutational changes in terms of biomolecular mechanism with relevance to human disease. 


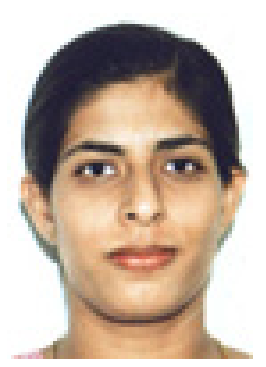

Durga Bhavani Kuchibhatla did her BSc in Computer Science at Osmania University, India and MSc in Bioinformatics at Nanyang Technological University, Singapore (2007). Then, after a stint at MDIS (Singapore) as a part-time lecturer, she joined the Bioinformatics Institute A*STAR in 2009. Her interests include developing sophisticated computer software for bioinformatics applications.

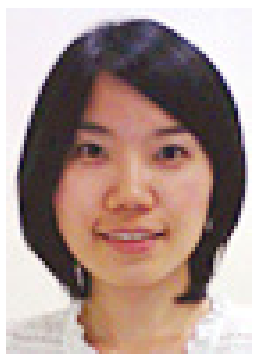

Vachiranee Limviphuvadh received her MSc in informational science from Graduate School of Informatics, and DSc from Graduate School of Science, Kyoto University, Japan, in 2001 and 2007, respectively. Meanwhile, she worked as an associate instructor and, later, as a postdoctoral research fellow in the Bioinformatics Center, Institute for Chemical Research, Kyoto University. Currently, she is a senior researcher in the Bioinformatics Institute, A*STAR, Singapore.

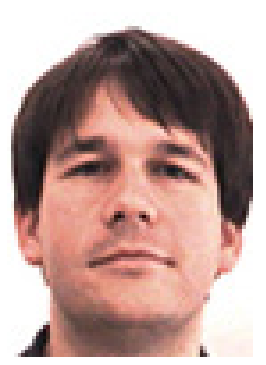

Sebastian Maurer-Stroh studied theoretical biochemistry at the University of Vienna and wrote his master and $\mathrm{PhD}$ thesis at the renowned Institute of Molecular Pathology (IMP). After the honor of a FEBS and a Marie Curie fellowship at the VIBSWITCH lab in Brussels, he leads a group of experts in protein sequence analysis in the Bioinformatics Institute (BII) since November 2007. He has contributed widely used predictors for posttranslational lipid modifications, amyloid fiber formation and catalyzed new biomedical insights by sequence-based function predictions. He has been at the forefront of research during the $2009 \mathrm{H} 1 \mathrm{~N} 1$ pandemic collaborating with hospitals and health authorities in Singapore, Mexico, Brazil, and Australia. 


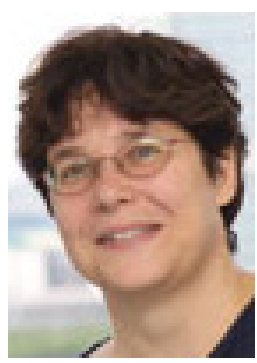

Birgit Eisenhaber's research interest is focused on the discovery of molecular functions of previously uncharacterized protein coding genes. The development of accurate prediction tools for proteins' posttranslational modifications and subcellular localization are one of the key points in her work. Birgit Eisenhaber studied biocybernetics and medicine at the Pirogov Medical University in Moscow. After working as a professional software developer, she restarted her scientific career at the EMBL Heidelberg. She received the $\mathrm{PhD}$ from the Humboldt University Berlin. Subsequently, she worked at the Institute of Molecular Pathology (IMP) Vienna and at the Experimental Therapeutics Centre (ETC) Singapore. In December 2010, Birgit Eisenhaber was appointed as a Principal Investigator at the Bioinformatics Institute (BII) Singapore.

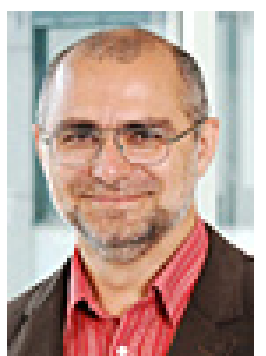

Frank Eisenhaber studied mathematics at the Humboldt-University in Berlin and biophysics and medicine at the Pirogov Medical University in Moscow (MD in 1985). He received the $\mathrm{PhD}$ in molecular biology from the Engelhardt Institute of Molecular Biology in Moscow 1988. After postdoctoral work at the Institute of Molecular Biology in Berlin-Buch (1989-1991) and at the EMBL in Heidelberg (1991-1999), he worked as team leader of the bioinformatics research group and head of the general IT department at the Institute of Molecular Pathology (IMP) in Vienna (1999-2007). Since August 2007, he is the Executive Director of the Bioinformatics Institute A*STAR Singapore. 